\title{
Similarities and dissimilarities in response to specific submaximal aerobic exercise in newcomer and well-trained athletes
}

\author{
Igor Anpilogov*, Pavel Pavlov \\ Kursk State University, 305000, Kursk, Russia
}

\begin{abstract}
The study was aimed on evaluation the applicability of certain distinct characteristics of heart rate dynamics dependence upon physical training load as criteria of young athletes functional fitness. Nine elite adult canoe and kayak rowers and twenty five young skiers aged 11-13 took part in the research program. Canoe and kayak rowers performed incremental tests on a rowing simulator $(4 * 1000 \mathrm{~m})$. The following parameters were measured: heart rate, oxygen heart rate, breath frequency, respiratory exchange ratio, oxygen uptake, and minute ventilation. Young skiers performed moderate run on a stadium track maintaining constant speed to voluntary physical exhaustion. Comparison of the trends in young and elite athletes allows assuming that the response to the given load on the third segment of the test exceeded the current level of fitness and continuation of the exercise at this level can cause overstrain and overtraining. It has to be pointed out that this assumption requires further research with measurement of a broader range of parameters.
\end{abstract}

\section{Introduction}

Finding novel methods and approaches to enhance efficiency of any age and level of sport qualification athletes training is a highly topical line of the contemporary sport science development [20].

According to scientific research results, the most efficient approaches to planning the athletic training process are based on the knowledge of behaviour of quantifiable parameters characterizing athlete's condition in response to various training programs $[10$, $12,21]$.

In young athlete training, implementation of a certain planning strategy has to take into consideration the age-specific dynamics of the trainees development and differences in responses to the same training stimulus between the young and the experienced, highly trained athletes $[6,9]$. However, to date most of the efforts to investigate adaptive responses of trainees to training exercise load are focused in elite athletes of various age groups and are mostly performed in the laboratory conditions $[3,14,18]$. Such studies offer deeper understanding of the physiological fundamentals of an athlete's body response to a training or testing load. However, in our opinion, they are of a limited value in defining biological

* Corresponding author: anpilogov.sport@yandex.ru 
criteria for assessment of training load's efficiency in young athletes in real training process.

Therefore, identification of the most informative methods of accessing the response to the training stimulus in young athletes aimed at preventing training overload and safely maximizing performance improvement is of special current interest. It is highly desirable that the proposed methods would not to significantly interfere with the training process, require no expensive scientific equipment.

We attempted to evaluate applicability of certain distinct characteristics of heart rate dynamics dependence upon physical training load as criteria of young athletes functional fitness.

\section{Material and methods}

Nine well-trained adult canoe and kayak rowers and twenty five young skiers aged 11-13 took part in the research program.

Canoe and kayak rowers performed incremental tests on a rowing simulator $(4 * 1000$ $\mathrm{m})$. The resting intervals between the stages of the test were equal to the duration of the immediately preceding segments performance. The last segment was performed with the maximal intensity. The following parameters were measured and evaluated: heart rate $(\mathrm{HR})$, oxygen heart rate(O2/HR), breath frequency (BF), respiratory exchange ratio (RER), oxygen uptake (VO2), and minute ventilation ( $\left.\mathrm{V}^{`} \mathrm{E}\right)$. The parameters were measured and recorded with the aid of Oxycon Mobile, Jaeger, Germany.

Young skiers performed moderate run on a stadium track maintaining constant speed to voluntary physical exhaustion. Garmin ForeRunner 230 was used to measure and record the heart rate, running speed and distance.

\section{Results and Discussion}

Our analysis and evaluation of the research results were based on the notion that the dynamics of adaptive changes in the body and its circulatory system, including in particular those determined by the specificity of the muscular activity, can be used as indicators of functional fitness $[2,5]$.

Dynamics of the "HR - speed" dependence has some individual particularities. They were reported by other researches [7, 13]. However certain generic trends can be identified. It should be noted that speed dynamics mostly depends upon the following two factors: the level of functional fitness and the mastery of the particular exercise technique. As the heart rate variations were relatively slow, we can assume that the mastery of running technique is the factor that limits the speed dynamics.

At the second stage, heart rate was stabilized. The running speed at this stage showed no common trends, but rather fluctuated in a wide range. We assume that lack of common trend in running speed is attributed to the existing broad variations in the level of young athletes technical skills. This stage, in our opinion, can be identified with the notion of a "short-term adaptation", a process in the course of which body is using the pre-existing physiological mechanisms to reach a relatively stable state (Meerson et al., 1988). Therefore, we may assume that speed and time of sustaining in this stage corresponds to the level of the athlete fitness. At the beginning of the test we observed rapid increase of speed and heart rate, the speed growth rate being significantly higher (Fig. 1). 


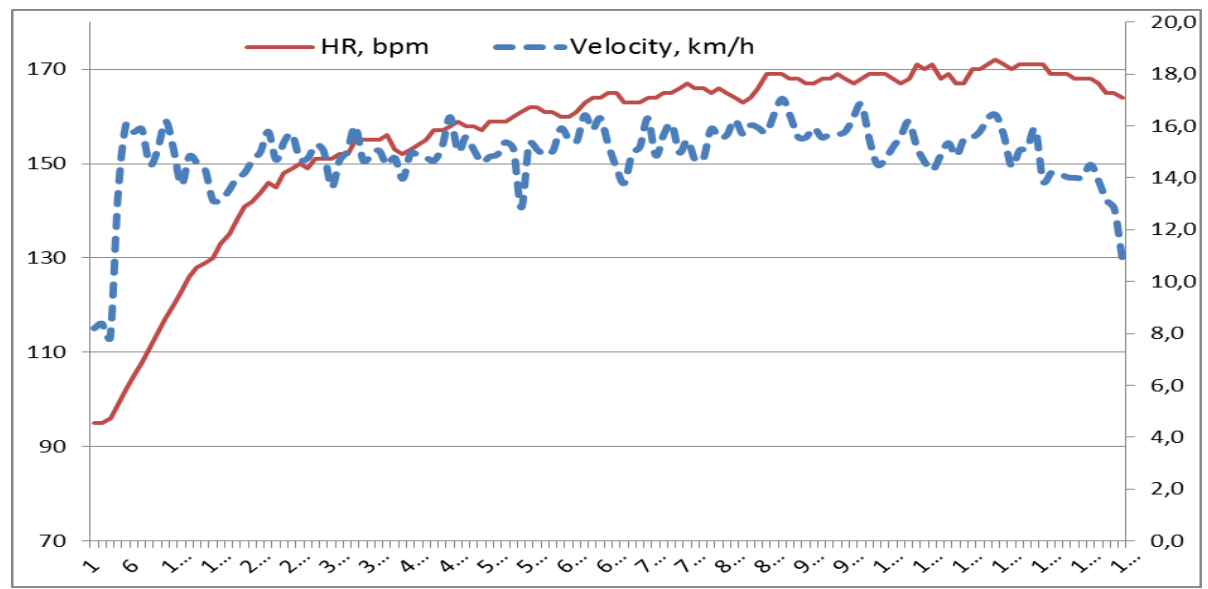

Fig 1. Dynamics of heart rate and speed in young athletes in the experiment

At the third stage of the test, the heart rate was observed to increase, while the running speed decreased substantially. Due to the fact, that the testing was done within regular training process without individualization of the testing speed for the voluntary physical exhaustion test, the third stage for the most of the participants began at HR above $180 \mathrm{bpm}$, and athletes at this stage abandoned the test almost immediately after reaching this stage. Accordingly, we assume that the level of functional changes in subjects' bodies were sufficiently above the anaerobic threshold level.

For better understanding of the functional changes at the last stage of a voluntary physical exhaustion test, we tested 9 adult elite canoe and kayak rowers. Taking into consideration that the level of functional fitness of elite athletes was sufficiently higher than the one of our young skiers, we selected incremental test to reach the full potential of the subjects. The length of each segment in the incremental test was equal to a competition distance $(1000 \mathrm{~m})$, the rest time was equal to the time on the previous segment. The first three segments were performed with sequentially growing intensity, the last one - at the maximal speed.

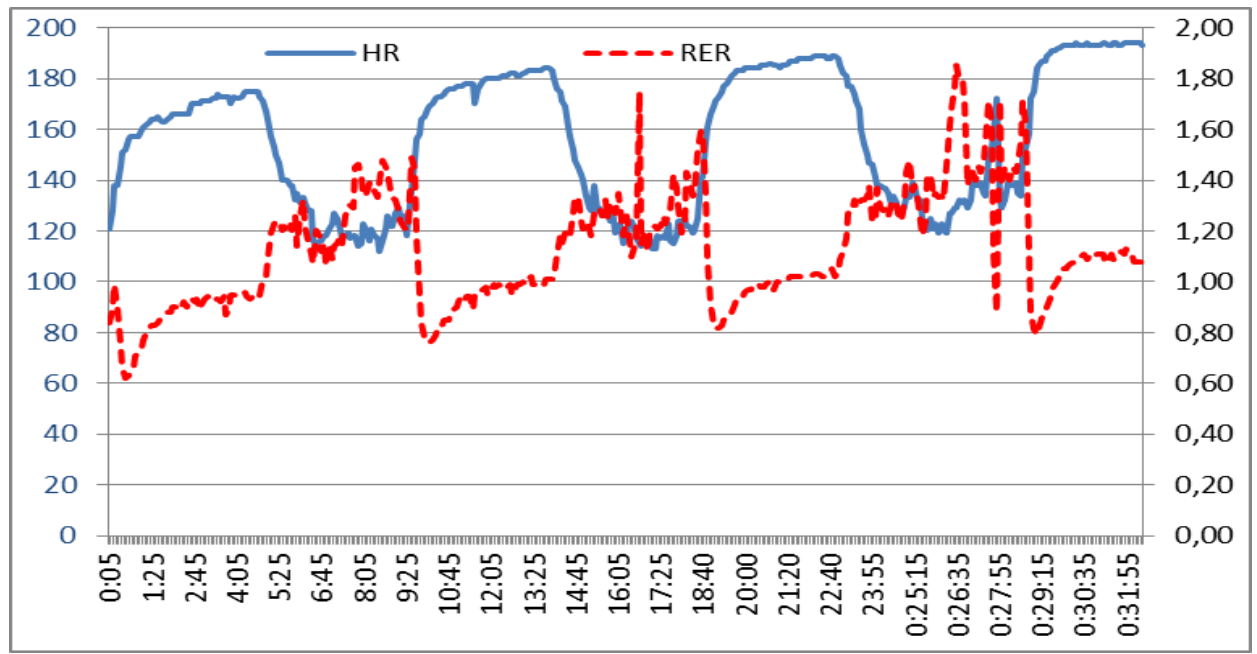

Fig. 2. The dynamics of heart rate and respiratory exchange ratio in elite athletes

All the subjects completed the first segment in the aerobic mode. RER was below one. At the end of a segment, all the measured parameters showed upward tendency. During the second and the third segments, athletes reached the anaerobic threshold level. It is worth 
mentioning that on the third segment the anaerobic threshold level was reached at higher levels of HR in comparison with the second segment. It is indicative of gradual recruitment of the athletes functional capabilities during exercises (Fig. 2).

The dynamics of the measured hemodynamic parameters on the last segment allowed us to state that the athletes reached critical level of their functional abilities. Thus the heart rate exceeded $190 \mathrm{bpm}$ and varied within a very narrow band of $+-2 \mathrm{bpm}$. Whereas the rest of the measured parameters were growing at the beginning of the segment, and then decreasing towards its end (Fig. 3-6).

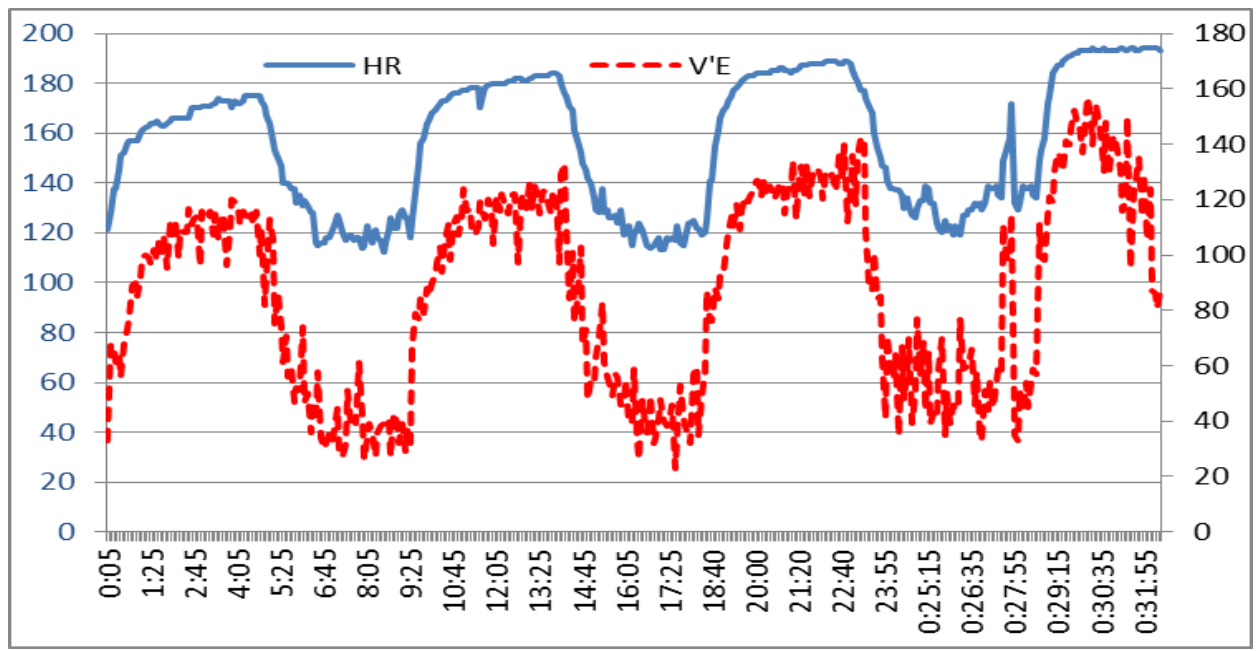

Fig. 3. Dynamics of elite athlete response to the workload during the incremental test

Decrease of the measured parameters at the last segment of the test were consistent with the evidence published by other researchers; in our opinion it indicated reduction of the cardiovascular and respiratory systems capacity to supply working muscles with oxygen [17].

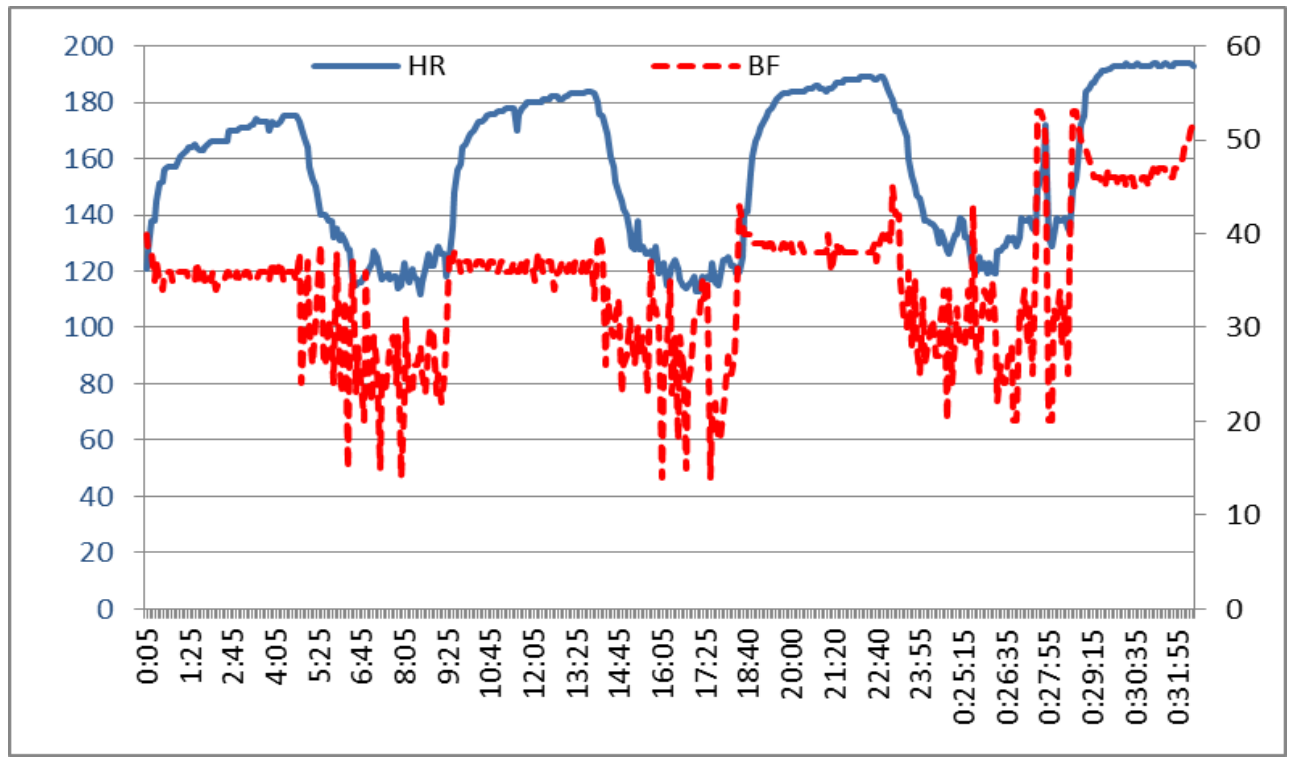

Fig. 4. Dynamics of elite athlete response to the workload during the incremental test 
Analysis of breath frequency dynamics during the test showed that increase of oxygen demand was supplied by increase of the breathing depth. However, at the last segment an increase of the breath circles number (Fig. 4) was compensated buy decreased depth of breathing (Fig. 3).

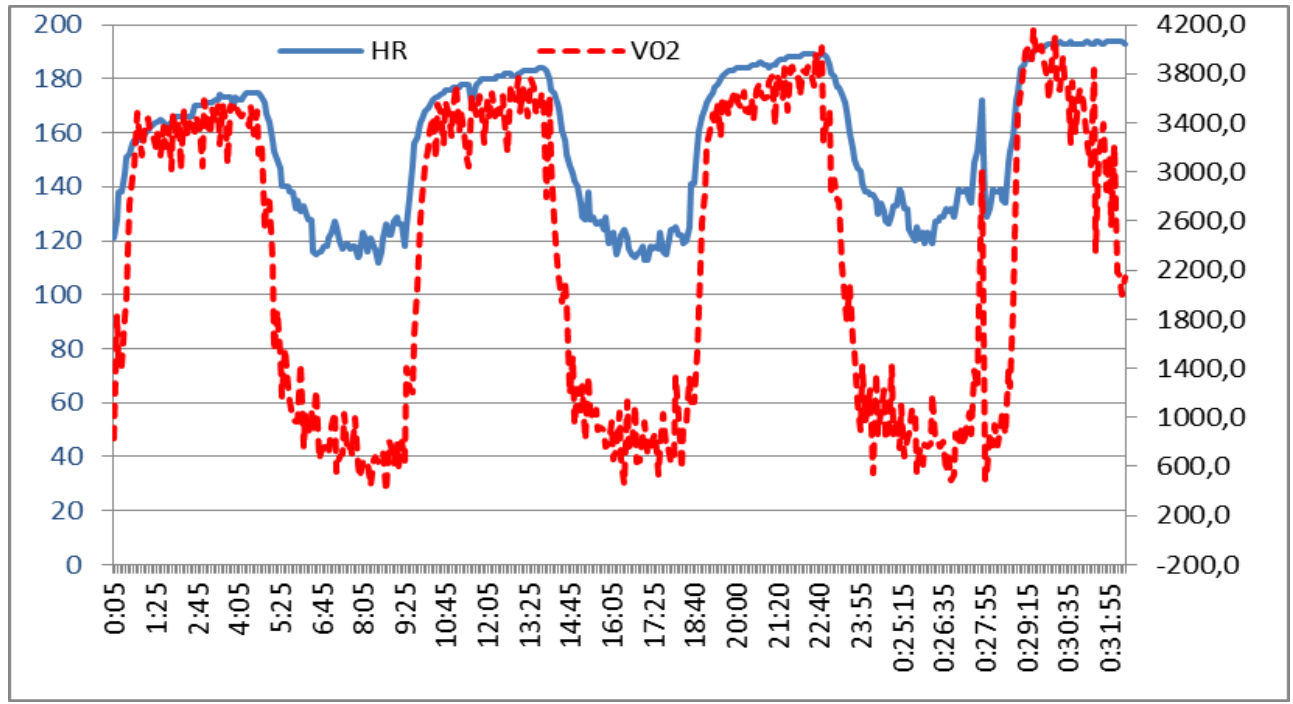

Fig. 5. Dynamics of elite athlete response to the workload during the incremental test

These changes resulted in reduction of the oxygen uptake (Fig. 5) and oxygen heart rate (Fig. 6).

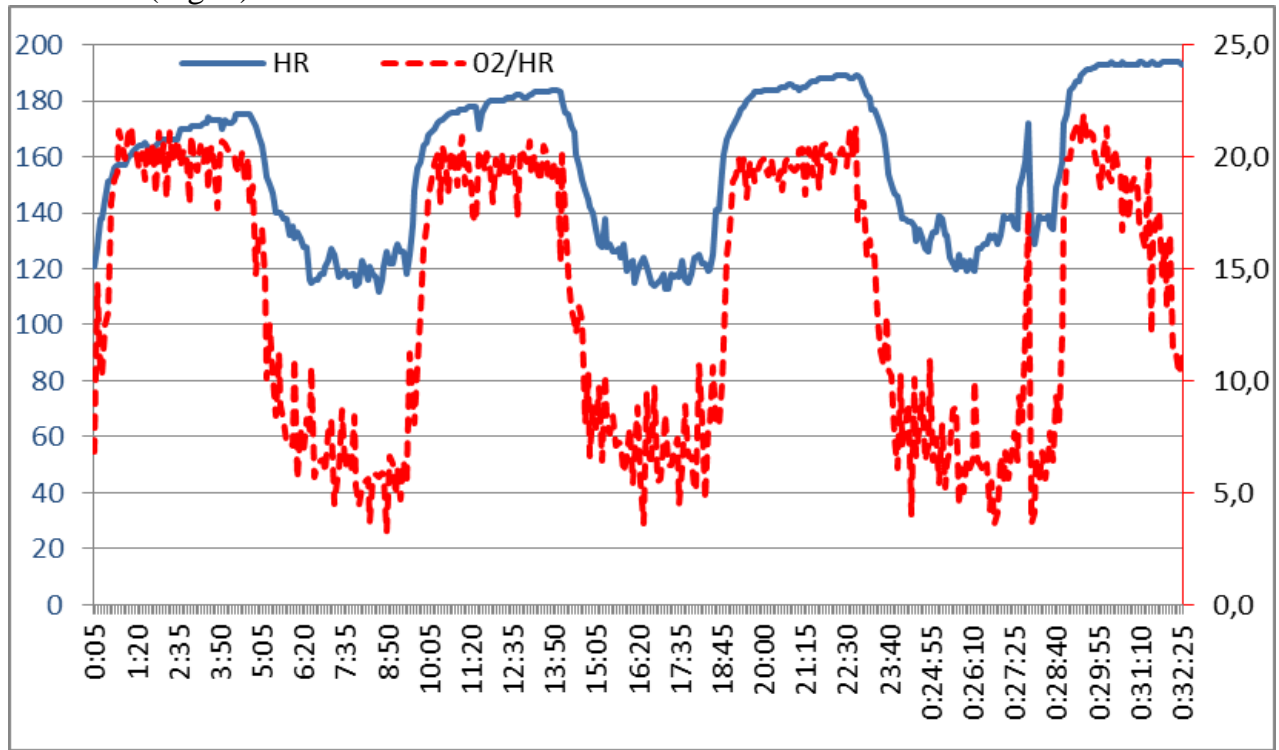

Fig. 6. Dynamics of elite athlete response to the workload during the incremental test

Therefore, the magnitude and the pattern of the measured parameters changes in elite athletes allowed us to conclude that workloads above the individual functional abilities, leads to decrease of work efficiency and rejection to continue the test.

At the same time, the level of functional fitness determines the work efficiency in the presence of increasing fatigue. The fact that all the athletes completed the last segment at 
high, but stable heart rate values, whereas all the rest of the measured parameters decreased significantly, supports this notion concept.

\section{Conclusions}

Comparison of the trends in young and elite athletes allows assuming that the response to the given load on the third segment of the test exceeded the current level of fitness and continuation of the exercise at this level can cause overstrain and overtraining.

It has to be pointed out that this assumption requires further research with measurement of a broader range of parameters. Nevertheless, we believe that the results we obtained so far significantly broaden understanding of the heart rate dynamics in young athletes in the course of natural training process and can, along with the outcome of further investigations, serve as a basis for development of efficient non-invasive methods for assessing functional fitness of young endurance athletes.

\section{Conflicts of interest}

The authors declare that there is no conflict of interest.

\section{References}

1. C.Y. Chen, S.E. DiCarlo, H.L. Collins, MSSE, 27(10), 1399-405 (1995)

2. O.V. Chertov, News of South Federal University, 12, 81-86 (2011)

3. L. Cipryan, G. Tschakert, P. Hofmann, JSSM, 16(2) (2017).

4. V.G. Coffey, H. Pilegaard, A.P. Gamham, B.J. O`Brian, J.A. Hawley, JAP, 106, 1187-1197 (2009).

5. F.A. Engel, A. Ackermann, H. Chtourou, B. Sperlich, Frontiers in Physiology, 9, 1012 (2018). DOI: https://doi.org/10.3389/fphys.2018.01012

6. B.W. Fudge, J. Wilson, C. Easton, L. Irwin, J. Clark, O. Haddow, B. Kayser, Y.P. Pitsiladis, MSSE, 39(1), 192-198 (2007). DOI: 10.1249/01.mss.0000235884.71487.21

7. R.L. Gellish, B.R. Goslin, R.E. Olson, A. Mcdonald, G.D. Russi, V.K. Moudgil, MSSE, 39(5), 822-829 (2007). DOI: $10.1097 / \mathrm{mss} .0 \mathrm{~b} 013 \mathrm{e} 31803349 \mathrm{c} 6$

8. P. Hofmann, S.P. Von Duvillard, F.-J. Seibert, R. Pokan, M. Wonisch, L.M. Lemura, G. Schwaberger. MSSE, 33(10), 1726-1731 (2009)

9. R.J. Iannotti, R.P. Claytor, T.S. Horn, R. Chen, MSSE, 36 (11), 1964-1971 (2004). DOI: 10.1249/01.MSS.0000145445.54609.82

10. V.F. Kostjuchenko, E.P. Vrublevsky, M.S. Kozhedub, STJUZUPFL, 10(152) (2017)

11. A.D. Mahon, C.S. Anderson, M.J. Hipp, K.A. Hunt, MSSE, 35(12), 2093-2097 (2003). DOI: 10.1249/01.MSS.0000099180.80952.83

12. P.R. Nader, R.H. Bradley, R.M. Houts, S.L. McRitchie, M. O'Brien, JAMA, 300(3), 295-305 (2008)

13. T. Otsuki, S. Maeda, M. Iemitsu, Y. Saito, Y. Tanimura, J. Sugawara, R. Ajisaka, T.I. Miyauchi. MSSE, 39(2), 365-370 (2007). DOI: 10.1249/01.mss.0000241647.13220.4c

14. P.V. Pavlov, I.V. Demin, PCSTMR, 5(1) (2020). DOI 10. 24411/ 2500- 0365- 2020- 15108

15. K. Roecker, A.M. Niess, T. Horstmann, H.; Striegel, F. Mayer, H.-H. Dickhuth, MSSE, 34(5), 881-887 (2002)

16. J.S. Skinner, S.E. Gaskill, T. Rankinen, A.S. Leon, D.C. Rao, J.H. Wilmore, C. Bouchard, MSSE, 35(11), 1908-1913 (2007). DOI: 10.1249/01.MSS.0000093607.57995.E3

17. S.J. Strath, A.M. Swartz, D.R. Bassett, W.L. O’Brien, G.A. King, B.E. Ainsworth, MSSE, 32(9), 465-S470 (2000) 
18. O. Tikkanen, S. Kärkkäinen, P. Haakana, M. Kallinen, T. Pullinen, T. Finni. MSSE, 46(9), 18311839 (2014). DOI: 10.1249/MSS.0000000000000298

19. E.P. Vrublevsky, S.V. Sevdalev, A.G. Narskin, MS. Kozhedub, Technology of individualization training of qualified athletes: theoretical and methodological aspects (Skoruna GSU; Gomel, 2016)

20. F. Zintel, A. Eisenhut, Ausdauertraining: grundlagen. methoden. Trainingssteuerung (BLV Sportwissen, München, 2009)

21. O.I. Kolomiets, N.P. Petrushkina, E.V. Bykov, I.A. Yakubovskaya, Pedagogico-psychological and medico-biological problems of physical culture and sports, 12(2), 217-225 (2017). DOI: 10.14526/01_2017_225. 